\title{
TOPOLOGY AND ELEMENTARY GEOMETRY. II. SYMMETRIES ${ }^{1}$
}

\section{H. GUGGENHEIMER}

Modern axiomatics of elementary geometry has steadily followed Hilbert's program of elimination of continuity arguments. A brilliant example of the success of this method is to be found in the book [1] by $\mathrm{F}$. Bachmann which gives a unified treatment for geometries over arbitrary fields of characteristic $\neq 2$. On the other hand the author has shown [2] that the plane axioms of incidence and order in Hilbert's system have very strong topological implications. Therefore it seems natural to invert Hilbert's procedure and to try and characterize euclidean (and hyperbolic) geometry by the topology of its plane and some continuity properties of isometric mappings. This is done in this note. We give first a topological formulation of the order axioms and deduce the Hilbert system from it. The topological approach allows some reduction of the axiom system. In the topological space generated we introduce symmetries. Since we dispose of a strong topological structure we can do with only part of Bachmann's axioms. Finally we derive the congruence axioms as theorems. The main results are that completeness of the uniform structure and the existence of an unique involutive automorphism for each line imply the three-symmetries-theorem and the existence of angle bisector and perpendicular bisector, i.e., of a transitive group of motions.

The topology of the plane $\pi$ depends in large measure on the cardinality $\boldsymbol{\aleph}_{\alpha}$ of $\pi$. For $\alpha=1$ the plane is the cartesian product of two real number lines, it is two-dimensional. For $\alpha>1$ it is totally disconnected and hence of dimension zero. Therefore it is interesting to note that we may put a cardinality axiom last in our list and never use it for the proof of congruence properties. This shows that the important topological properties in plane geometry are completeness and connectedness but not arcwise connectedness and dimension.

We shall freely use the language of elementary geometry where no confusion can result.

1. The plane $\pi$ is a set whose elements, the points, are denoted by capitals. Certain subsets of $\pi$ are "lines," they are denoted by lower case Roman letters or by the symbols of the points defining them.

I. Axioms of incidence.

Received by the editors July 31, 1962 and, in revised form, October 27, 1962.

1 This research was supported by the Air Force Office of Scientific Research. The author is indebted to the referee for helpful remarks. 
I.1. To two distinct points there exists a unique line of which both are elements.

I.2. There exists a line.

II. Axioms of order.

II.1. The complement in $\pi$ of a line is the union of two disjoint nonvoid sets.

These sets are the halfplanes of the line. The halfplanes of a line $l$ will often be denoted by $1_{l}$ and $2_{l}$.

Definition 1. $C$ is between $A$ and $B$, written $(A C B)$, if $C \in A B$ and if there exists a line $c$ through $C$ (i.e., $C \in C$ ) such that $A$ and $B$ are in different halfplanes of $c$. The segment $(A B)$ is the set of all points $X$ between $A$ and $B$.

II.2. $P_{1}$ and $P_{2}$ are in different halfplanes of a line $l$ if and only if $\left(P_{1} P_{2}\right) \cap l \neq \varnothing$. (Pasch's axiom.)

II.3. $(A B C)$ implies non- $(A C B)$.

Proposition 1. A line contains at least one point.

To $l$ (I.2) there exists $A \in 1_{l}$ and $B \in 2_{l}$ (II.1) hence also $C=A B \cap l$ (II.2).

Proposition 2. There exist three noncolinear points.

With the notations of the previous proof there exists $D \in 1_{\Delta B}$. $A, B, D$ are not colinear, hence distinct (I.1).

Propositions 1 and 2 are usually taken as incidence axioms.

Proposition 3. ( $A C B$ ) implies $(B C A)$.

The definition is symmetric in $A$ and $B$.

Lemma 1. A line contains at least two points.

By Proposition 1, a line $g$ contains a point $A$. By Proposition 2 there exist $B$ and $C$ not colinear with $A$. If either $B \in g$ or $C \in g$ the lemma holds. If $B$ and $C$ are in different halfplanes of $g, X=B C \cap g$ exists (II.2) and is distinct from $A$. If $B$ and $C$ are both in the same halfplane there exists $D$ in the other one (II.1). Put $Y=B D \cap g$, $Z=C D \cap g$. Either $Y \neq Z$, or $Y=Z$ but $Y \neq A$ since then $B, C$, and $Y$, are colinear.

Definition 2. A point $A$ on a line $l$ is of first kind if it is the intersection of $l$ with a line $P Q$ where $P \in 1_{l}, Q \in 2_{l}$. A point is of second kind if it is not of first kind.

Proposition 4. Let be $A, B \in l$. If $B$ is of first kind there exists an $X$ such that $(A B X)$. If $B$ is of second kind there exists a $Y$ such that $(A Y B)$ but no $X$ such that $(A B X)$. A line has at most two points of 
second kind. If $B$ is of second kind on $l$, it is of second kind on any line to which it belongs.

If $B$ is of first kind and $A \in 1_{P Q}$ there exists $C \in 2_{P Q}$. Either $C=X$ or, if $C \notin l$, by II.3 one and only one of the sets $(C P) \cap l,(C Q) \cap l$ is nonvoid and can serve as $X$.

If $B$ is of second kind, assume that $X$ exists. $Q \in 1_{l}$ defines a line $g=Q B$. For $R \in 2_{l}, R$ is in one of the halfplanes of $g$ since $R \notin g$. But then by II.3 either $(R A) \cap g$ or $(R X) \cap g$ is a point $S \in 2_{\imath}$ and $B \in Q S$ contrary to assumption.

It follows immediately that Lemma 1 can be amended to read: "A line contains at least two points of first kind," hence a $Q \in 1_{A B}$ which exists by II.1 may be taken as of first kind. The usual proof (e.g. [2, Proposition 8]) that of three points one is between the other two then carries over to our system of axioms. Hence a line has at most two points of second kind.

If $A$ is of first kind, the proof on page 6 of the 8th edition of Hilbert's Grundlagen carries over to show that there exists $Y,(A Y B)$. If $A$ and $B$ are of second kind, the two points of Lemma 1 must be between $A$ and $B$.

The last statement of the proposition is immediate.

REMARK. The use of axiom II. 3 cannot be eliminated from this proof. There exists a model of axioms I.1, I.2, II.1, II.2 which consists of 5 points and 6 lines. Then Proposition 4 cannot hold. There also exists a model which shows that I.1, I.2, II.1, II.2, and Proposition 4 , do not imply II.3.

An analysis of the preceding proof shows that the following holds:

THEOREM 1. For any model of a geometry satisfying axioms I.1, I.2; II.1, II.2, II.3, the set of points of the first kind with the induced definition of lines forms a maximal submodel in which axioms I.1 to II.3 and

II.4. To $A, B$, exists $X$ such that $(A B X)$ hold.

In the terminology of [2], II.4 is a postulate rather than an axiom• In [2] an axiom system was discussed based on I.1, I.2, II.1, II.2, II.3, II.4, Proposition 1, Proposition 2, Proposition 3. The halfplanes as a basis of the open sets generate a topology which is normal, uniformizable, and may be completed in a unique way such as to conserve the betweenness relation. Therefore we add

II.5. The halfplane topology of $\pi$ is complete.

All results of [2] may be used for our system of axioms. If the cardinality of $\pi$ is $\aleph_{\alpha}$ all lines are ordered sets similar to a totally ordered, complete, dense in itself, abelian group $R_{\alpha}$ of cardinality $\aleph_{\alpha}$. $\pi$ has a faithful model in a convex set $S \subset R_{\alpha} \times R_{\alpha}$ of bounded co- 
ordinates [2, Theorem 9]. The boundary points of $S$ in $R_{\alpha} \times R_{\alpha}$ are in one-to-one correspondence to the classes of mutually parallel lines [2, Theorem 8, Lemmas 5,6].

2. III. Axioms of Symmetry.

III.1. To each line l there exists a mapping $\sigma_{l}$ of $\pi$ into itself different from the identity map $\iota$.

III.2. $\sigma_{l} \sigma_{l} P=P$ for all $l$ and all $P \in \pi$.

III.3. $\sigma_{L} P=P$ if $P \in l$.

III.4. $\sigma_{\imath}$ maps halfplanes into halfplanes.

III.5. A product of symmetries $\sigma_{a}$ which leaves a line $l$ pointwise fixed is either $\sigma_{l}$ or $\iota$ (Hjelmslev's Axiom 3).

Definition 2. $g$ is perpendicular to $l(g \perp l)$ if $\sigma_{l} g=g$ but $g \neq l$.

LEMMA 2. $\sigma_{l}$ is one-to-one and onto $\pi$.

By III. $2 \sigma_{l}$ is an involution.

LEMMA 3. $\sigma_{l}$ is continuous.

The inverse image of an open set is its image (III.2) and is open (III.4).

Lemma 4. $\sigma_{l}$ maps lines into lines.

By III.4 and Lemma 2, $\sigma_{l}$ maps the complement of the two halfplanes of a line into the complement of the union of two disjoint halfplanes.

Lemma 5. $(A B C)$ implies $\left(\sigma_{l} A \sigma_{l} B \sigma_{l} C\right)$.

Follows from the definition, III.4, and Lemma 4.

Lemma 6. If $A \neq \sigma_{\nu} A$ then the segment $\left(A \sigma_{\nu} A\right)$ contains a fixed point.

$B_{1} \in\left(A \sigma_{l} A\right)$ implies $\sigma_{l} B_{1} \in\left(A \sigma_{l} A\right)$ (Lemma 5 ). It is possible to choose names such that $A B_{1} \sigma_{l} B_{1} \sigma_{l} A$ be an ordered sequence [2, Theorem 1 and III.2]. By (transfinite) induction it is possible to select an ordered sequence $\left\{B_{\xi}\right\}_{\xi<\omega_{\alpha}}$ such that $\left(B_{\mu} \sigma_{l} B_{\mu}\right) \subset\left(B_{\nu} \sigma_{l} B_{\mu}\right)$ for $\mu>\nu$. The step from $\xi$ to $\xi+1$ is obvious. If $\xi$ is a limit number, take $B_{\xi} \in \bigcap_{\tau<\xi}\left(B_{\tau} \sigma_{l} B_{\tau}\right)$. The sequence $\left\{B_{\xi}\right\}$ is bounded by all $\sigma_{l} B_{\xi}$. It is possible to proceed so that no segment is contained in all $\left(B_{\mu} \sigma_{l} B_{\mu}\right)$. By completeness there exist a limit point which must be fixed under $\sigma_{l}$.

Proposition 5. $\sigma_{l} P=P$ only if $P \in l$.

Assume $\sigma_{l} Q=Q$ for some $Q \notin l$ and let be $P \in l$. $(P Q)$ is mapped into itself by $\sigma_{l}$ by III.3 and Lemma 5. By Lemma 6 applied to a point 
in $(P Q)$ and its image it follows that $(P Q)$ contains a fixed point distinct from $P$ and $Q$. Repeating the argument one obtains a dense set of fixed points on all segments $(P Q)$. Also, $\{X \mid(X Q P)\}$ is mapped into itself by $\sigma_{l}$. Therefore it contains a dense set of fixed points by the previous argument. The same holds true for $\{X \mid(X P Q)\} . \sigma_{l}$ is the identity on a set everywhere dense in $\pi$, Lemma 3 implies a contradiction to III.1.

Corollary 1. If $A \notin l$ then $\left(A \sigma_{l} A\right) \cap l \neq \varnothing$. $\sigma_{l}$ permutes the halfplanes of $l$. If $\sigma_{l}$ leaves two points fixed on some $g$, then $g=l$.

Proposition 6. Through any point $P$ there exists a unique perpendicular to a given line $l$.

a. $P \notin l . P \neq \sigma_{l} P$ by Proposition 5. The line $P \sigma_{l} P$ is mapped into itself by $\sigma_{l}$, hence $P \sigma_{l} P \perp l$. Any perpendicular to $l$ through $P$ must contain $\sigma_{l} P$, hence it is $P \sigma_{l} P$ by I.1.

b. $P \in l$. Assume first that there are two distinct perpendiculars $p_{1} \perp l$ and $p_{2} \perp l$ through $P \in l$. Choose $X \in p_{1}$ and $Y \in p_{2}$ in the same halfplane $1_{l}$. Then $Y$ and $\sigma_{l} Y$ are in different halfplanes of $p_{1}$ by II.2 since $\left(Y P \sigma_{l} Y\right)$ holds. Hence $\left(X \sigma_{l} Y\right) \cap\left(Y \sigma_{l} X\right)=\varnothing . Q=\left(X \sigma_{l} Y\right)$ $\in l$ and $R=\left(Y \sigma_{l} X\right) \cap l$ exist by Corollary 1 and II.2. By the previous argument $Q \neq R$. But $\sigma_{l}\left(X \sigma_{l} Y\right)=\left(\sigma_{l} X Y\right)$ by Lemma 5 , hence $Q=R$ by III.3. Contradiction.

The existence of a perpendicular through $P$ follows by a completeness argument similar to that used for Lemma 6. On a line $g \neq l$ through $P$ choose $X$ and $Y$ in different halfplanes. This is always possible. If $g$ is not perpendicular to $l$ we choose $B_{1} \in\left(X \sigma_{l} Y\right)$. Let $B^{*}=\sigma_{l} B_{1} P \cap\left(X \sigma_{l} Y\right)$, it exists by II.2. It is possible to choose names such that $X B_{1} B_{1}^{*} \sigma_{l} Y$ is an ordered sequence. As before one may construct a Cauchy $\omega_{\alpha}$-sequence $\left(B_{\mu} B_{\mu}^{*}\right)_{\mu<\omega_{\alpha}}$ such that $\left(B_{\rho} B_{\rho}^{*}\right) \subset\left(B_{\sigma} B_{\sigma}^{*}\right)$ for $\rho>\sigma$. For the limit point $B=B^{*}$ it follows that $\sigma_{l} B P=B P$ and $B P \neq l$.

LEMMA 7. $\sigma_{\sigma_{a} b}=\sigma_{a} \sigma_{b} \sigma_{a}$.

For $Q \in \sigma_{a} b$ one has $\sigma_{a} \sigma_{b} \sigma_{a} Q=Q . \sigma_{a} \sigma_{b} \sigma_{a} \neq \iota$ since $\sigma_{b} \neq \iota$. The lemma follows from III.5.

LEMMA 8. $b \perp a$ if and only if $\sigma_{a} \sigma_{b}=\sigma_{b} \sigma_{a}$ and $a \neq b$.

If $b \perp a$ then $\sigma_{\sigma_{a} b}=\sigma_{a} \sigma_{b} \sigma_{a}=\sigma_{b}$.

If $\sigma_{a} \sigma_{b}=\sigma_{b} \sigma_{a}$ then $\sigma_{a}$ leaves $\sigma_{b} a$ pointwise fixed. Hence $\sigma_{b} a=a$. The lemma follows by definition.

Corollary 2. $a \perp b$ implies $b \perp a$. 
Proposition 7. $a \perp b$ implies $\sigma_{c} a \perp \sigma_{c} b$ for all $c$.

$$
\sigma_{\sigma_{c} a} \sigma_{\sigma_{c} b}=\sigma_{c} \sigma_{a} \sigma_{c} \sigma_{c} \sigma_{b} \sigma_{c}=\sigma_{c} \sigma_{b} \sigma_{a} \sigma_{c}=\sigma_{\sigma_{c} b} b \sigma_{\sigma_{c} a}
$$

REMARK. Minkowski geometry in the plane can be used to construct models which show that Lemma 7 and Proposition 7 depend essentially on axiom III.5. A strengthened form of Proposition 7 together with the other axioms implies III.5; see [1, \$2.4, Satz 3].

Lemma 9. Two distinct concurrent lines $a$ and $b$ have no common perpendicular.

Let $p$ be a common perpendicular. If $P=a \cap b \notin p$, contradiction to Proposition 6. If $P \in p$ then by Corollary 2, $a \perp p, b \perp p$, contradiction to Proposition 6.

Lemma 10. If $a, b$ are distinct concurrent lines then $\sigma_{a} \sigma_{b}$ has exactly one fixed point.

$P=\sigma_{a} \sigma_{b} P$ is fixed. If $Q=\sigma_{a} \sigma_{b} Q$ for $Q \neq P$ then $\sigma_{a} Q=\sigma_{b} Q \neq Q$ and $l=Q \sigma_{a} Q$ is a common perpendicular to $a$ and $b$. Then use Lemma 9 .

A point $P$ on a line $l$ divides $l$ into two rays $l_{1}$ and $l_{2}$. The ray from $P$ which contains $Q$ will also be denoted $(P) Q$. By II.2 one may take any line $g \neq l$ through $P$ and define $l_{1}=l \cap 1_{g}, l_{2}=l \cap 2_{g}$. Two rays $l_{1}$ and $k_{1}$ issuing from the same point $P$ form an angle $\Varangle\left(l_{1}, k_{1}\right)$. The interior of the angle is the intersection $1_{l} \cap 1_{k}$ of the halfplanes of $l$ containing $k_{1}$ and of $k$ containing $l_{1}$. An angle bisector of $\Varangle\left(l_{1}, k_{1}\right)$ is a line $b$ such that $\sigma_{b} l_{1}=k_{1}$. By Lemma 6 an angle bisector must have a ray in the interior of the angle. For three rays $a_{1}, b_{1}, c_{1}$, starting from $P$ we write $\left(a_{1}, b_{1}, c_{1}\right)$ if $b_{1}$ is in the interior of $\Varangle\left(a_{1}, c_{1}\right)$. The linear order of rays can be reduced to that of points $[2, \S 4]$.

LeMMA 11. In any finite product of symmetries $\Sigma$ angle bisectors are mapped into angle bisectors.

By Lemma 7, $\sigma_{b} l=k$ implies $\sigma_{\Sigma b} \Sigma l=\Sigma k$.

3. The treatment of metric planes in [1] is based not in III.5 but on the much stronger statement:

Theorem 2. A finite product $\Sigma$ of symmetries which maps a ray $l_{1}$ onto itself is either $\sigma_{l}$ or $\iota$.

The proof of the theorem follows different paths in the euclidean and noneuclidean cases. The latter is simpler due to the absence of homothecies. Since I need some parallel axiom in the proof of Theorem 2 which itself is needed to establish the existence of a transitive group of motions, up to now I am unable to decide whether there exist 
geometries with I.1-III.5 and not uniformly euclidean or hyperbolic. However, I am able to show that Axioms I, II, III, and either one of the statements of Axiom V, Proposition 8, or Proposition 9, imply that IV.H is equivalent to the negation of IV.E. This will be the subject of a separate paper.

An end is an equivalence class of first parallels. For our purposes the most convenient formulation of the hyperbolic axioms is

IV.H. Two distinct ends define a line in $\pi$.

This line is unique. Otherwise for lines through points in the strip bounded by two lines with identical endpoints at least for one direction the second end is determined by the first, in contradiction to axiom IV.H.

An end can be represented by a ray that belongs to it. Let $d$ be the perpendicular from $P=l \cap k$ to the line $l_{1} k_{1}$. Then $\sigma_{d} l_{1}=k_{1}$ since $\sigma_{d}$ exchanges halfplanes, conserves perpendicularity and maps ends into ends. The last statement follows from Lemma 3 and the construction of parallels by a limit process. Any bisector $d^{*}$ of $l_{1}$ and $k_{1}$ maps $l_{1} k_{1}$ and $P$ onto themselves, hence also $\sigma_{d^{*}}\left(d \cap l_{1} k_{1}\right)=d \cap l_{1} k_{1}$ and $d^{*}=d$ by Corollary 1.

Proposition 8. Two rays have a unique angle bisector.

For two distinct points $P_{1} Q$ let be $p$ and $q$ the perpendiculars in $P$ and $Q$, respectively, to $P Q$. Then $\sigma_{P Q p_{1}} q_{2}=p_{2} q_{1}$, hence $X=p_{1} q_{2} \cap p_{2} q_{1}$ $\in P Q$. In terms of ends $p_{1} \neq q_{1}$ since otherwise $p_{1} p_{2}$ would define more than one line. Let be $x$ the perpendicular from $X$ to $p_{1} q_{1}, x$ is a perpendicular bisector of $P Q$, i.e., $\sigma_{x} P=Q$. By construction, $\sigma_{x} p_{1}=q_{1}$, $\sigma_{x} X=X$, hence by Corollary $2 \sigma_{x} P Q=P Q$ and $\sigma_{x}(P Q \cap) p=P Q \cap q$. As for the angle bisector one shows uniqueness.

Proposition 9. Two points have a unique perpendicular bisector.

Proof of Theorem 2. Without loss of generality one may assume that $\Sigma$ conserves halfplanes and that $(P \Sigma X X)$ for all $X \in l_{1}$ where $P \in l$ defines $l_{1}$ (eventually replace $\Sigma$ by $\sigma_{l} \Sigma$ or $\Sigma^{-1}$ ). We show that $\Sigma$ has a dense set of fixed points. Let be $d$ the bisector of $l_{1} k_{1}, D=d \cap l_{1} k_{1}$. Then $\Sigma D=D$.

(a) Between two fixed points $H, K$ of $\Sigma$ on $l_{1}$ there exists a third one, e.g. the midpoint (Proposition 9).

(b) In the order on $l_{1}$ there is no last fixed point. Let $F$ be fixed, $f \perp l$ through $F$ is mapped into itself by $\Sigma$. Let $g$ be the angle bisector of $l_{1}$ and $P f_{1} . g$ is invariant by Lemma 11 and Proposition $8, g \cap f_{1} l_{1}$ exists and is fixed. Its orthogonal projection $R$ onto $l_{1}$ is fixed and satisfies $(P F R)$. 
(c) Let $\left(H_{\lambda} K_{\lambda}\right) \lambda<\mu<\omega_{\alpha}$ be a nested sequence of intervals defined by fixed points of $\Sigma$. The endpoints of the closure of $\cap\left(H_{\lambda} K_{\lambda}\right)$ must be fixed. This completes the proof.

4. Next, we assume

IV.E. Through a point not on a line there exists (at most) one parallel to the line.

We retain the previous conventions about $\Sigma, l_{1}, k_{1}$, and $P$. Since parallels are perpendicular to a common transversal (Proposition 6), parallels are transformed into parallels by $\Sigma$.

Proposition 9 holds for IV.E. Let $p, q$ be the perpendiculars to $P Q$ in $P$ and $Q$. For any $X \in p_{1}$ let $Y$ be the projection of $X$ onto $q_{1}$. Define $T=X Q \cap P Y$ (exists by II.2) and $t \perp P Q, T \in t$; and let $U=t \cap P Q$. Then $\sigma_{t} P Q=P Q$. If $\sigma_{t} P \neq Q$ one may assume $\left(U \sigma_{t} P Q\right)$, eventually by a change of names. $\sigma_{t} Q$ is the projection onto $P Q$ of $\sigma_{t} Y=\sigma_{t} P T \cap X Y$, hence $\left(P \sigma_{t} Q U\right)$ or $\left(\sigma_{t} P Q U\right)$, contradiction. $t$ is the perpendicular bisector.

If $t$ and $u$ are bisectors, then necessarily $u \perp P Q$ and by construction, $\sigma_{u} T=T$, hence $t=u$.

As before it follows that $\Sigma$ is the identity if it has two fixed points.

Next we prove as a first step towards Proposition 8: Angle bisectors are unique. If $a$ and $b$ are bisectors of $l_{1}$ and $k_{1}$, so is $\sigma_{a} b$. Let $M$ be the midpoint of $X$ and $\sigma_{b} X$ for some $X \in l_{1}, M^{*}$ that of $\sigma_{a} X$ and $\sigma_{a} \sigma_{b} X$. Hence $M M^{*} \perp a . X$ and $\sigma_{a} X$ are in different halfplanes of $M M^{*}$, hence $X \sigma_{a} X \cap M M^{*} \neq \varnothing$ is a point of $a$. But this implies $M M^{*}$ $=X \sigma_{a} X, \sigma_{a} X=\sigma_{b} X$ and $a=b$.

As a consequence one has

LEMMA 12. A ray cannot be invariant in $a$ map $\sigma_{a} \sigma_{b}$ if $a \neq b$.

Proposition 8 can now be proved. The circle of center $P$ and radius $(P A)$ is the set of all images $\sigma_{b} A$ for fixed $A$ and $b \ni P$. The order of rays in $P$ induces an order of points on the circle. We shall take care to represent symmetry lines by their rays $b_{1}$ in the halfplane $1_{P A}$. $\sigma_{b} A$ is a strictly monotone function of $b$, i.e., $\left(P A b_{1} b_{1}^{\prime}\right)$ implies $\left(A \sigma_{b} A \sigma_{b}, A\right)$ on the circle. Since the angle bisector is unique, any ray through $P$ has at most one point in common with the circle. Assume that $\left(A \sigma_{b}, A \sigma_{b} A\right)$ holds. Define $p_{0}=P A, p_{\lambda+1}=\sigma_{b} \sigma_{b}, p_{\lambda} ; q_{0}=b^{\prime}, q_{\lambda+1}$ $=\sigma_{b} \sigma_{b^{\prime}} q_{r}$. For limit numbers choose interior $\Varangle\left(p_{\lambda}, q_{\lambda}\right) \subset \bigcap_{\tau<\lambda}$ interior $\chi\left(p_{\tau}, q_{\tau}\right)$ such that the order is always $\left(p_{\lambda} p_{\mu} q_{\mu} q_{\lambda}\right)$ for $\mu>\lambda$, and that no angle is in the interior of all $\chi\left(p_{\lambda} q_{\lambda}\right)$. By completeness there exists a ray $q$ such that $\sigma_{b} \sigma_{b}, q$ against Lemma 12 . It now follows easily by a completeness argument that the circle has one point in 
common with each ray and that the bisector exists.

If $\Sigma$ leaves a ray invariant, then so do the perpendiculars to $l$ through $P_{1}$ and the angle bisectors of $l$ and $k$, and their bisectors (Lemma 11) and so on. Since $\Sigma$ must be continuous on the rays issuing from $P$, one constructs a dense set of invariant rays. $\Sigma$ must leave all rays through $P$ invariant. If $\Sigma \neq \iota, \Sigma$ may have no fixed point other than $P$ (Corollary 1 ), hence any line $g$ not passing through $P$ is mapped into a parallel to $g$ (the point $X=g \cap \Sigma g$, if it exists, is mapped into $\Sigma X \in P X$, i.e., $X=\Sigma X$ or $P \in g$ ).

Let $\Sigma=\sigma_{a(k)} \sigma_{a(k-1)} \cdots \sigma_{a(1)}$ where all $a^{(i)}$ are concurrent at $P$. Then Theorem 2 holds. It holds for $k=1$. By the preceding argument, $\sigma a_{1}^{(1)}=a_{1}^{(1)}$ if we assume that $\Sigma$ preserves halfplanes. If Theorem 2 holds for $k-1$ then $\sigma_{a(k)} \cdots \sigma_{a(2)}=\iota$ or $\sigma_{a(1)}$, hence $\Sigma=\iota$ or $\sigma_{a(1)}$. The second case is excluded since $\Sigma$ preserves halfplanes.

Proposition 10. For three concurrent lines $a, b, c$ there exists $d$ such that $\sigma_{a} \sigma_{b} \sigma_{c}=\sigma_{d}$.

Let $d$ be the angle bisector of $c_{1}$ and $\sigma_{a} \sigma_{b} c_{1}$. Then $\sigma_{d} \sigma_{a} \sigma_{b} \sigma_{c}=\imath$ or $\sigma_{c}$. The second case is impossible, $\sigma_{a} \sigma_{b} \neq \sigma_{d}$ by Lemma 10.

Let $\Sigma=\sigma_{a(k)} \cdots \sigma_{a(1)}$ where all $a^{(i)}$ are perpendiculars to a line $l$. Then Theorem 2 holds. Let $\Sigma P=P$, and let $p \perp l$ through $P$. Since any perpendicular to $p$ is transformed into itself by all $\sigma_{a(i)}$ (IV.E and Corollary 2), $p$ is pointwise fixed under $\Sigma, Q . E . D$.

As a consequence of the same argument

LEMma 13. If $a$ and $b(a \neq b)$ are both perpendicular to $a$ line $l$ then $\sigma_{a} \sigma_{b}$ has no fixed point, since $\sigma_{a} \sigma_{b}=\sigma_{p}$ would imply $\sigma_{a} \sigma_{b}=\sigma_{b} \sigma_{a}$ or $a \perp b$.

Proposition 11. To three distinct lines $a, b, c$ perpendiculars to $a$ line $l$ there exists $d$ such that $\sigma_{d}=\sigma_{a} \sigma_{b} \sigma_{c}$.

$d$ is the perpendicular bisector of $C \in c$ and $\sigma_{a} \sigma_{b} C . \sigma_{d} \sigma_{a} \sigma_{b}=\iota$ is excluded by Lemma 13.

It follows that any $\Sigma$ is the product of not more than three symmetries. By Lemmas 10 and 13, a $\Sigma$ appearing in Theorem 2 is the product either of one or of three symmetries. The only case remaining is $\Sigma=\sigma_{a} \sigma_{b} \sigma_{c} ; a, b, c$ neither concurrent nor parallel and $P \notin a \cup b \cup c$. No generality is lost if we assume that $Q=b \cap c$ exists. Since $\Sigma P Q$ $=P \sigma_{a} Q$ it follows that $Q \in P \sigma_{a} P$ and from $\sigma_{a} P=\sigma_{b} \sigma_{c} P$ and Lemma 12 that $\left(P Q \sigma_{a} P\right)$. By the same lemma, since $\sigma_{b} \sigma_{c} \sigma_{b} \sigma_{c}(Q) P=\sigma_{b} \sigma_{\sigma_{c} b}(Q) P$ $=(Q) P, b$ and $c$ are perpendicular. In this case $\sigma_{b} \sigma_{c} a=a$ since $a$ is the perpendicular bisector of $P=\left(\sigma_{b} \sigma_{c}\right)^{2} P$ and $\sigma_{b} \sigma_{c} P$. This implies the invariance of $R=P Q \cap a$ under $\sigma_{b} \sigma_{c}$, hence $Q=R \in a$, the three lines are concurrent against hypothesis. Theorem 2 is completely proved. 
5. The Hilbert axioms of congruence now become theorems on symmetries.

DEFINITION 3. Two sets $S$ and $S^{\prime}$ are congruent $\left(S \cong S^{\prime}\right)$ if there exists a product of symmetries $\Sigma$ such that $\Sigma S=S^{\prime}$.

C.1. To any segment $(X Y)$ and any ray $a_{1}$ starting from a point $A$ there exists $B \in a_{1}$ such that $(A B) \cong(X Y)$.

$B=\sigma_{d} \sigma_{m} Y$ where $m$ is the perpendicular bisector of $(A X)$ and $d$ the angle bisector of $<\left(a_{1}, \sigma_{m} X Y\right)$.

C.2. The congruence relation is transitive.

C.3. If $(A B C),\left(A^{\prime} B^{\prime} C^{\prime}\right),(A B) \cong\left(A^{\prime} B^{\prime}\right), \quad(B C) \cong\left(B^{\prime} C^{\prime}\right)$, then $(A C) \cong\left(A^{\prime} C^{\prime}\right)$.

$A^{\prime} B^{\prime}=\Sigma A B, \quad B^{\prime} C^{\prime}=\Sigma^{*} B C . \quad\left(A^{\prime} B^{\prime} \Sigma C\right)$ and $\left(\Sigma^{*} A B^{\prime} C^{\prime}\right)$ imply $\Sigma^{-1} \Sigma^{*}(B) C=(B) C$. By Theorem $2, \Sigma=\Sigma^{*}$ or $\Sigma=\sigma_{A B} \Sigma^{*}$. In both cases, $\Sigma C=C^{\prime}$.

C.4. To any angle $\Varangle\left(h_{1}, k_{1}\right)$ and any ray $h_{1}^{\prime}$ there exists $k_{1}^{\prime}$ in either halfplane of $h^{\prime}$ such that $\Varangle\left(h_{1}, k_{1}\right)=<\left(h_{1}^{\prime}, h_{1}^{\prime}\right)$.

Proof like that of C.1.

C.5. $\Varangle\left(h_{1}, k_{1}\right) \cong \Varangle\left(k_{1}, h_{1}\right)$.

Symmetry at angle bisector.

C.6. Let $A_{1} B_{1} C$ be three noncolinear points. If $(A B) \cong\left(A^{\prime} B^{\prime}\right)$, $(A C) \cong\left(A^{\prime} C^{\prime}\right)$ and $\Varangle C A B \cong \Varangle C^{\prime} A^{\prime} B^{\prime}$ then $\Varangle A B C \cong \Varangle A^{\prime} B^{\prime} C^{\prime}$.

There exist $\Sigma$ such that $\Sigma(A B)=\left(A^{\prime} B^{\prime}\right)$ and $\Sigma C \in$ half plane of $A^{\prime} B^{\prime}$ containing $C^{\prime}$. Also there exists $\Sigma^{\prime}, \Sigma^{\prime}(A C)=\left(A^{\prime} C^{\prime}\right)$ and mapping the halfplane of $B$ into that of $B$ ! Finally there is $\Sigma^{*}$ with maps rays: $\Sigma^{*}(A) B=\left(A^{\prime}\right) B^{\prime}, \Sigma^{*}(A) C=(A) C^{\prime}$. By Theorem $2, \Sigma^{-1} \Sigma^{\prime}=\Sigma^{-1} \Sigma^{*}=\imath$. Hence $\Sigma C=C^{\prime}$ and $\Varangle A B C=\Varangle A^{\prime} B^{\prime} C^{\prime}$. This shows:

THEOREM 3. Euclidean and hyperbolic geometry are the only geometries which satisfy axioms I.1,2; II.1,2,3,4,5; III.1,2,3,4,5, IV.H or IV.E, and V.

The cardinality of $\pi$ is the continuum.

The "propositions" proven have been chosen so that Bachmann's axioms in [1, p. 24 (33), 201, 216 (also p. 126, Satz 2)] can be verified immediately.

\section{REFERENCES}

1. F. Bachmann, Aufbau der Geometrie aus dem Spiegelungsbegriff, Springer, Berlin, 1959.

2. H. Guggenheimer, The topology of elementary geometry, Math. Japon. 5 (1959), 1-26.

3. J. Hjelmslev, Einleitung in die allgemeine Kongruenzlehre. Erste Mitteilung, Mat.-Fys. Medd. Danske Vid. Selsk. (11) 8, (1927-9).

UNIVERSITY OF MINNESOTA 